\title{
Local Structural Damage Evaluation of a C/C-SiC Ceramic Matrix Composite
}

\author{
Benedicta D. Arhatari, ${ }^{1, *}$ Matthew Zonneveldt, ${ }^{2}$ John Thornton, ${ }^{2}$ and Brian Abbey ${ }^{1}$ \\ ${ }^{1}$ ARC Centre of Excellence in Advanced Molecular Imaging, Department of Chemistry and Physics, La Trobe Institute for \\ Molecular Science, La Trobe University, Bundoora, VIC 3086, Australia \\ ${ }^{2}$ Air Vehicles Division, Defence Science and Technology Group, Port Melbourne, VIC 3207, Australia
}

\begin{abstract}
Ceramic matrix composites (CMCs) are structural materials, which have useful properties that combine high strength at high temperatures with moderate toughness. Carbon fibers within a matrix of carbon and silicon carbide, called $\mathrm{C} / \mathrm{C}-\mathrm{SiC}$, are a particular class of $\mathrm{CMC}$ noted for their high oxidation resistance. Here we use a combination of four-point bending and $\mathrm{X}$-ray radiography, to study the mechanical failure of $\mathrm{C} / \mathrm{C}$-SiC CMCs. Correlating X-ray radiographic and load/displacement curve data reveals that the fiber bundles act to slow down crack propagation during four-point bending tests. We attribute this to the fact that strain energy is expended in breaking these fibers and in pulling fiber bundles out of the surrounding matrix material. In addition, we find that the local distribution and concentration of SiC plays an important role in reducing the toughness of the material.
\end{abstract}

Key words: composite, tomography, X-rays, mechanical test, microstructure

\section{INTRODUCTION}

Ceramic matrix composites (CMCs) have been studied extensively due to their ability to combine high strength, moderate toughness, and low density while still exhibiting very good temperature resistance (Krenkel, 2008; Genet et al., 2012). They are thus ideally suited for applications in extreme environments such as within the hot-sections of gas turbine engines in aircraft (Krenkel et al., 2002). Furthermore, some CMCs have high wear resistance and high resistance with respect to aggressive chemicals. These distinctive properties are the result of the materials microstructure which is composed of a ceramic matrix and ceramic fibers (Krenkel, 2008). As their name suggests CMCs contain ceramic fibers embedded in ceramic matrices, for example carbon fibers within a matrix of carbon and silicon carbide ( $\mathrm{C} / \mathrm{C}-\mathrm{SiC}$ composites). They are designed to achieve damage tolerant, quasi-ductile fracture behavior. At the same time, CMCs retain many of the other desirable properties of monolithic ceramics such as excellent creep resistance and high stiffness (Raether, 2013). The advantage of CMCs over pure monolithic materials (e.g., solid graphite) are that more elastic energy is required in a CMC to propagate cracks. This is due to the fact that additional energy is expended in separating the matrix material from the fibers (Bale et al., 2013). The amount of energy consumed will increase in proportion to the strength of bonding between the fibers and surrounding matrix. However, if the bonding becomes too strong then it becomes more energetically favorable for the crack to propagate by travelling through the

Received August 4, 2016; accepted March 23, 2017

*Corresponding author. B.Arhatari@latrobe.edu.au fibers rather than by separating them from the host matrix. In this case, the composite behaves more like a monolithic ceramic. Ideally, heating and reaction during CMC manufacture should not cause too strong bonding between matrix and fiber, as this would lead to a decrease in the effective toughness.

CMC toughness mainly depends on the fiber architecture, fiber content, manufacturing process, and the composition of the materials used to protect against oxidation (Krenkel, 2008). The progressive degradation of CMC structures used for high temperature applications has been reported in previous studies (Bale et al., 2013). Degenerative effects on both the fiber scale, for example fiber oxidation, or on the composite scale, for example intra- and inter-tow degradation have been observed (Genet et al., 2012). The carbon-carbon family of CMCs maintain their mechanical strength up to extremely high temperatures $\left(3,000^{\circ} \mathrm{C}\right)$, however they oxidize rapidly at moderate temperatures $\left(500^{\circ} \mathrm{C}\right)$ in air. In the present case, in order to protect the $\mathrm{CMC}$ from oxidation and enable the composite material to operate at higher temperatures, $\mathrm{SiC}$ was added to the CMC. The mechanical properties of carbon/carbon materials have been investigated in a number of previous studies. However, less is known about how these mechanical properties are modified when $\mathrm{SiC}$ is added to the matrix to improve oxidation resistance (Roy et al., 2014). Also, there are few studies on how any of these modified properties are related to the microstructure. This lack of detailed information linking microstructure to performance has motivated the present study of $\mathrm{C} / \mathrm{C}-\mathrm{SiC} \mathrm{CMC}$.

The direct correlation of X-ray radiography images, collected during in situ mechanical loading to a materials load response, can offer a unique insight into its properties. 
Using this approach, damage evolution processes such as crack initiation and growth (Bale et al., 2013; Dassios et al., 2014), or other failure mechanisms (Zhu et al., 2012), can be monitored as they occur. In previous work, the sequence of damage cracking mechanisms in composites was investigated using X-rays (Nouri et al., 2013). The authors showed that the use of X-ray imaging enables a deeper understanding of the proposed failure mechanisms for these types of materials. Such information can then be used to reduce the risk of failure during their operational lifetime, as well as allowing for the optimization of their performance. In addition, micro X-ray computed tomography ( $\mu \mathrm{XCT})$ is a powerful and well-established imaging tool that has the ability to image structures in three dimensions at high spatial resolution (Awaja \& Arhatari, 2009; Abbey et al., 2010; Baimpas et al., 2014). The basic principle of $\mu \mathrm{XCT}$ is that the sample is irradiated with X-rays, while X-ray absorption contrast images are recorded as a function of sample rotation with respect to the source and detector. The series of images can then be combined and reconstructed for full threedimensional (3D) visualization of the sample (Kak \& Slaney, 1988). $\mu$ XCT can thus be used to look for any anisotropy within the $\mathrm{CMC}$ fiber microstructure, the distribution of $\mathrm{SiC}$ (and unreacted $\mathrm{Si}$ ) within the $\mathrm{C} / \mathrm{C}-\mathrm{SiC}$ matrix, and the presence of voids or cracks. This $3 \mathrm{D}$ information contributes to a deeper understanding of the limits of strength, toughness and oxidation resistance of the material (Korsunsky et al., 2010; Pedrazzini et al., 2016). For example, in areas where there are gaps in the SiC network it is possible to infer that the oxidation resistance of the structure will be low and this type of information can easily be determined via X-ray tomography.

In previous work (Bale et al., 2013), X-ray imaging was used to characterize the microstructure of CMCs during loading. The study found that the local CMC microstructure plays a key role in its failure (Czabaj et al., 2014; Frazer et al., 2015; Sloof et al., 2016). In the present study, we apply similar techniques to investigate how the microstructure influences the mechanical behavior of a $\mathrm{C} / \mathrm{C}$ (carbon fiber with carbon matrix) CMC impregnated with SiC. High-resolution, X-ray radiographic imaging data were collected during in situ four-point bend mechanical loading of the $\mathrm{C} / \mathrm{C}-\mathrm{SiC}$ CMC sample. To enable a more in-depth interpretation of the radiography results, complimentary measurements were made using $\mu \mathrm{XCT}$ after failure had occurred, as well as backscattered electron microscopy of the broken surface. By combining high-resolution X-ray and electron imaging with in situ mechanical testing, we aimed to characterize the failure mechanisms for this sample and asses the relative influence of the local distribution of $\mathrm{SiC}$.

\section{Materials and Methods}

\section{Sample}

A C/C-SiC CMC, provided by the Institute of Structures and Design, German Aerospace Center in Stuttgart, was used for this study. The CMC was produced by infiltration of molten silicon into a porous $\mathrm{C} / \mathrm{C}$ preform. This was followed by heating (at around $1,600^{\circ} \mathrm{C}$ ) to enable the $\mathrm{SiC}$ network to be formed from the reaction of the silicon with some of the carbon matrix (Heidenreich, 2012). This process is called liquid silicon infiltration (LSI); the associated CMC produced via LSI is referred to here as 2DLSI. The 2DLSI samples have a characteristic strength-to-weight ratio and are particularly well-suited to high temperature applications.

The 2DLSI samples studied here consisted of two directional bundles of carbon fibers in a carbon matrix encased within a network of SiC. The internal SiC network provided additional oxidation protection for the $\mathrm{C}-\mathrm{C}$ material via an inert protective layer of $\mathrm{SiO}_{2}$ (Raether, 2013; Roy et al., 2014). Four samples of 2DLSI were used for four-point bend testing labeled as "2DLSI_" followed by "A," "B," "C," and "D." The thickness $(b)$ and height $(d)$ of samples 2DLSI_A, 2DLSI_B, 2DLSI_C, and 2DLSI_D were $1.45 \times 3.00,1.38 \times 3.00,1.38 \times 3.00$, and $1.20 \times 3.00 \mathrm{~mm}^{2}$ $(b \times d)$, respectively. This defines the cross-section of each of the samples. We note that some variation in the sample thickness occurs during preparation of specimens for testing. However, this thickness variation is taken into account in our calculations. Hence, the thickness variation in the samples is not expected to influence the results presented here. For comparison, we also used three samples of a unidirectional $\mathrm{C} / \mathrm{C}-\mathrm{SiC} \mathrm{CMC}$ and three samples of pure graphite labeled "1DPI_" and "Graphite_," respectively, followed by "A," "B," and "C."

The 1DPI samples studied here consisted of one directional bundle of carbon fibers encased in a phenolic resin matrix. The resin underwent pyrolysis to convert it to carbon. A silicon containing polymer was then infiltrated into the matrix before a final round of pyrolysis to convert this polymer into $\mathrm{SiC}$. The thickness and height of unidirectional samples 1DPI_A, 1DPI_B, and 1DPI_C were $1.90 \times 2.08$, $1.76 \times 1.92$, and $1.91 \times 2.33 \mathrm{~mm}^{2}(b \times d)$, respectively.

The third material studied was high purity, artificial isotropic graphite (Selaby Australia Pty. Ltd., Carrum Downs, Victoria, Australia). Graphite is a solid monolithic ceramic, which does not contain any fibers nor SiC. The thickness and height of graphite samples Graphite_A, Graphite_B, and Graphite_C were $1.25 \times 3.21,1.23 \times 3.21$, and $1.25 \times 3.21 \mathrm{~mm}^{2}(b \times d)$, respectively.

\section{Loading Device}

A compact in situ loading rig (CT500N; Deben Ltd., Woolpit, Bury Saint Edmunds, UK) capable of both tensile and compressive testing was used for the four-point bending tests. The motor speed that determined the rate of loading was set to $0.2 \mathrm{~mm} / \mathrm{min}$. The maximum available load for this device is $500 \mathrm{~N}$, while the load accuracy is $1 \%$. The loading rig was mounted on the sample stage within the $\mathrm{X}$-ray tomography instrument, enabling recording of X-ray radiographs during mechanical loading experiments. A schematic 

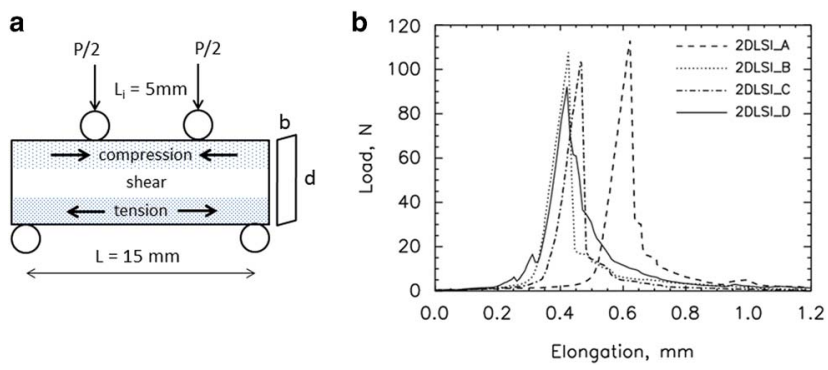

Figure 1. a: Schematic diagram of four-point bend test. It should be noted that the direction of the incident X-ray beam is out of the page. b: Load versus elongation plot for the four-point bend experiment of all four 2DLSI samples.

diagram of the four-point bend test is shown in Figure 1a. During four-point bending (following the ASTM D6272-10 method) ${ }^{a}$ radiography images were continuously recorded at $1-$ s time intervals.

\section{$\mathrm{X}$-ray Imaging}

An $\mathrm{Xradia}^{\odot}$ micro XCT200 (Carl Zeiss X-ray Microscopy, Inc., Pleasanton, CA, USA) was used for all X-ray imaging. This includes both the $\mu \mathrm{XCT}$ measurements and X-ray radiography data collected during mechanical testing. The XCT200 uses a microfocus X-ray source with a rotating sample holder and a high-resolution imaging detector system. The source consists of a closed X-ray tube containing tungsten as a target material. For this experiment, we set the operating tube voltage to be $40 \mathrm{kV}$ giving a peak power of $10 \mathrm{~W}$. The imaging detector comprised an X-ray scintillator optically coupled to an Andor CCD camera (Oxford Instruments, South Windsor, CT, USA) via an objective lens that could be changed depending on the desired resolution and field of view (FOV). In the present case, a $2 \times$ objective lens was used for the $\mathrm{X}$-ray radiography imaging and a $4 \times$ objective lens was used for the $\mu \mathrm{XCT}$ imaging. The two different X-ray set-ups are explained as follows:

1. The first set-up was designed to acquire a series of radiography images during the four-point bend experiment; the effective sample pixel size was $17.5 \times 17.5 \mu \mathrm{m}$ (image size $512 \times 512$ pixels) with a FOV of $8.9 \times 8.9 \mathrm{~mm}$.

2. The second set-up was designed to acquire $\mu \mathrm{XCT}$ data after sample failure with an effective sample voxel size of $3.5 \times 3.5 \times 3.5 \mu \mathrm{m}$ and a corresponding FOV of $3.6 \times 3.6 \times 3.6 \mathrm{~mm}$. One $\mu \mathrm{XCT}$ data set consisted of 721 equiangular projections over $180^{\circ}$, which provided a complete tomographic reconstruction. The exposure time was $60 \mathrm{~s} /$ projection.

To minimize beam-hardening artifacts, which can occur when using polychromatic $\mathrm{X}$-ray sources, a beam-hardening correction was applied during the reconstruction process.

\footnotetext{
${ }^{a}$ ASTM D6272-10, Standard test method for flexural properties of unreinforced and reinforced plastics and electrical insulating materials by fourpoint bending, ASTM International, West Conshohocken, PA, USA, 2010, www.astm.org.
}

The beam-hardening corrected intensity is described by the following expression (Zou et al., 2011)

$$
\ln \left(\frac{I_{0}}{I_{\text {corr }}}\right)=\ln \left(\frac{I_{0}}{I_{\text {meas }}}\right)+A\left[\ln \left(\frac{I_{0}}{I_{\text {meas }}}\right)\right]^{2} \ln \left(\frac{I_{0}}{I_{\text {meas }}}\right),
$$

where $I_{\text {meas }}$ is the measured projection intensity with the sample in place, $I_{0}$ the reference intensity without the sample, and $I_{\text {corr }}$ the corrected projection intensity. Equation (1) assumes a second-order relationship for the polynomial "correction factor." A filtered back projection algorithm (using the commercial TXMReconstructor software package; Carl Zeiss X-ray Microscopy, Inc., USA) was used to reconstruct a $3 \mathrm{D}$ image of the sample. The reconstruction software allowed for the selection of an optimum value for the constant $A$ based on the quality of the reconstructed image. For the present experiment, a value of $A=0.5$ for all the projection images was found to produce artifact-free three-dimensional reconstructions. The three-dimensional reconstructed image size was $1024 \times 1024 \times 1024$ voxels. The commercial rendering package, TXM3DViewer (Carl Zeiss X-ray Microscopy, Inc., USA), was used for $3 \mathrm{D}$ visualization and to generate the final images presented in this paper.

\section{Volume Segmentation Analysis and Scanning Electron Microscopy}

Volume segmentation, postreconstruction, was carried out using the Avizo-6.2 software package (Mercury Computer Systems Inc. Mérignac, Bordeaux, France). This process used the three-dimensional reconstructed data from the $\mu \mathrm{XCT}$ experiment to calculate both the $\mathrm{SiC}$ and $\mathrm{C}$ content. Automated segmentation was performed by local thresholding of the different gray-scale levels based on the absorption histogram and inspection of the reconstructed images (Pal \& Pal, 1993), described in the Supplementary Figure 1.

\section{Supplementary Figure 1}

Supplementary Figure 1 can be found online. Please visit journals.cambridge.org/jid_MAM.

The segmentation process, which was used to discriminate between the carbon (matrix and fibers) and the $\mathrm{SiC}$, excluded the surrounding air, which represents the background outside of the sample. Visual inspection of the reconstructed images, aided in the selection of an appropriate threshold, was then used to differentiate features in the histogram (Supplementary Fig. 1). The SiC and carbon could be readily distinguished from each other based on their different microstructure (boxes versus fibers). Scanning electron microscopy (SEM) showed that there was a considerable number of $\mathrm{SiC}$ particles that were both smaller than the $\mu \mathrm{XCT}$ voxel size and also dispersed throughout the fiber bundles. The limited resolution of the $\mu \mathrm{XCT}$ images and the polychromatic nature of the X-ray source, thus made determining an exact threshold value in the segmentation process 
challenging. This is due to the fact that the $\mathrm{SiC}$ particles, which are not properly resolved, take on a gray level in the images which is in between that of carbon and SiC. In addition, the residual effects of beam hardening may have also caused some voxels to be incorrectly assigned to either $\mathrm{SiC}$ or carbon. By incrementing the gray level in the histogram, we established a lower and upper bound for the $\mathrm{SiC}$ content which was used to establish the uncertainties on the volume fractions quoted in the text.

Backscattered electron microscopy was carried out using a high-performance scanning electron microscope (SEM) (JSM-6490LA; JEOL, Akishima, Japan). The SEM was fitted with an X-Max 80 detector (X-Max; Oxford Instruments, Tubney Woods, Abingdon, UK) which allowed for energy-dispersive X-ray measurements to be performed. A backscattered electron image produces information about the sample surface morphology and was used to characterize the fracture surface after failure. The electron microscopy images have a resolution of the order of $1 \mu \mathrm{m}$ and provide complementary data to support the X-ray analysis.

\section{Results}

\section{Four-Point Bend Experiment}

The load versus elongation curve for the four 2DLSI specimens, collected during four-point bend testing, is displayed in Figure $1 \mathrm{~b}$. We examined the direction of crack propagation during the test to determine whether the crack was caused by flexure or shear. In most cases, the path of the crack was in the vertical direction (perpendicular to the tension load direction in Figure 1a), indicating that flexure was the dominant mechanism responsible for crack propagation. The flexural stress of the specimens was calculated as follows

$$
\sigma=\frac{3}{2} P \frac{\left(L-L_{i}\right)}{b d^{2}},
$$

where, $P$ is the applied load, $b$ the sample thickness, $d$ the sample height, $L$ the span between the lower supports, $L_{i}$ the load span. The maximum flexural stress before failure of the four 2DLSI specimens was measured as 123, 129, 124, and $129 \mathrm{MPa}$, respectively. This is at the lower end of the scale based on previously published failure tests of C/C-SiC CMCs (Krenkel, 2008) which we attribute to the specific composition of $\mathrm{SiC}$ and manufacturing used for the present sample. Previous studies of the strength of C/C-SiC CMCs, however, have not looked in detail at the relationship between microstructure and the failure properties of the samples.

In Figure 1b, the four 2DLSI samples all have a similar peak shape. However, there are important differences in the amount of energy required to bend the samples further. To clarify these differences, we first classify the different regions and features using the load plots of 2DLSI_A in Figure 2. We then discuss how some of the regions and features vary between the different samples. The radiographs in this figure correspond to the points on the load plot, P1 to P4. The load plots of all the samples start with an initial flat region (I).

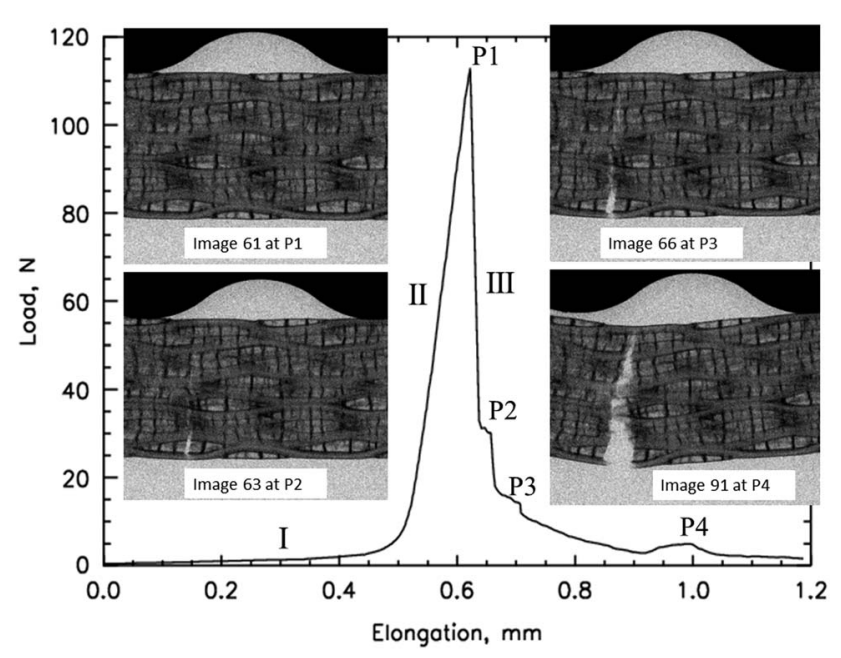

Figure 2. The load plot of 2DLSI_A with the corresponding radiographs of points $\mathrm{P} 1-\mathrm{P} 4$.

This corresponds to the slack being taken-up in the loading device. Its length is unimportant, as each sample will have been set up with a slightly different degree of slack. Steep rises (II) follow the flat region in all of the load plots. These steep rises correspond to the sample resisting the motion of the four-point bend fixtures. Eventually, the load becomes large enough to initiate a crack at the bottom of the sample and the resistance starts to decrease. Just before the sample cracks (image 61 in Fig. 2) the load plot reaches a maximum (P1). This maximum (P1) is followed by a sudden drop in the stress (III) which accounts for the majority of crack growth. However, the rate of decrease in region (III) varies between samples. For 2DLSI_A, the drop in the load is very sudden which corresponds to the rapid progress of the crack through the relatively brittle material. In contrast, for 2DLSI_D, the decrease is more gradual corresponding to slower crack propagation through an effectively tougher material. In the tail section of 2DLSI_A's plot, there is an additional small peak (P4) which we attribute to the crack attempting to progress through bundles of fibers perpendicular to the average plane of the crack and parallel to the tensile load at the crack tip. The increase of the peak heights relates to tensioning of the fiber bundles and the storage of elastic energy. While the decrease of the peak heights correspond to breaking of the fibers and pull out of the bundles from the surrounding matrix (Budiansky \& Hutchinson, 1986).

The peak's position in the tail of the load plot is due to the fact that these fiber bundles are at the edge of the sample and at the end of the crack (image 91 in Fig. 2). 2DLSI_A also contains two smaller peaks (P2 to P3). Similar to P4, these peaks correspond to pullout of fiber bundles perpendicular to the crack plane. However, from the radiographic images, P2 and P3 are associated with pullout of fibers from the middle of the sample. These bundles are left bridging the crack after the bulk of the crack tip has already passed them by (images 63 and 66 in Fig. 2). There are some other slight differences between the load curves for the different samples. 


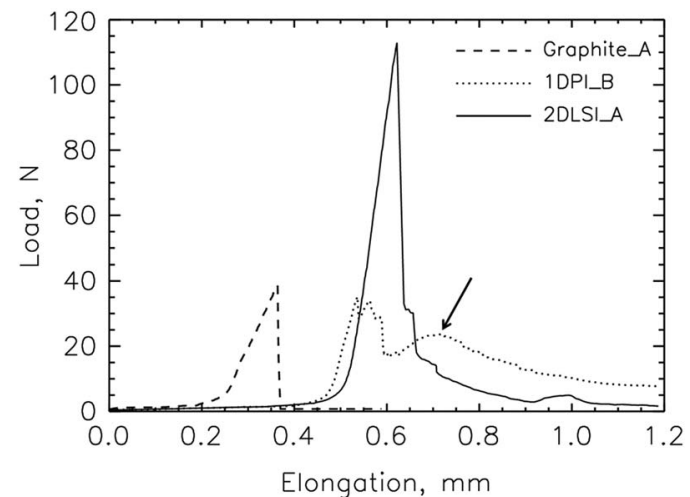

Figure 3. The comparison load plots for 2DLSI_A, Graphite_A and 1DPI_B sample.

For example, 2DLSI_D's plot shows some small peaks at the start of the major rise. These correspond to the pullout of fiber bundles at the bottom of the sample which is also the origin of the crack.

Overall though, the main features in the plots are similar. It should be noted that the radiographs show that the crack propagated quickly through the majority of the 2DLSI_A sample (image 63 in Fig. 2). However, the sample remained held together by bundles of fibers over a large elongation, from P2 to P3. The work done by in situ loading in this region is consumed by pulling these bundles from the matrix or by breaking the fibers within them. The radiographs show that the size of the crack opening increased with the extraction of the fiber bundles.

Figure 3 compares the load plots of 2DLSI_A with Graphite_A and 1DPI_B. The area under the load curves, which is normalized by:

$$
\frac{3}{2} \frac{\left(L-L_{i}\right)}{b d^{2}}
$$

corresponds to the energy required to break the sample. A small area indicates a brittle material while a large area is characteristic of a tough material. The load plot for a brittle material will exhibit a sharp drop (III) as shown for Graphite_A in Figure 3. The load plot for a tough material will exhibit a gradual drop (III) as shown for the unidirectional C/C-SiC 1DPI_B sample. The area up to the maximum load corresponds to the energy stored in elastic deformation of the sample. The elastic energy stored, and the additional energy applied during the gradual drop, corresponds to the energy required to break the material or drag fibers from the matrix, as well as generating heat and motion within the matrix. A comparison of the area under the load curves is given in Table 1. Additional integration of the area up to the next maximum load point in the gradual drop region (indicated by the arrow in Fig. 3) has been used to calculate the area for unidirectional samples, since the samples can still resist the increasing load. We note that these calculations assume that the sample height, $d$, is constant up to the last peak in the load plot. In Table 1, the same types of sample are grouped together and the standard deviation
Table 1. Comparison of the Area Under the Load Curves and Maximum Flexural Strength for Three Different Types of Samples, i.e. Graphite, 2DLSI, and 1DPI Samples.

\begin{tabular}{lcccc}
\hline & \multicolumn{3}{c}{$\begin{array}{c}\text { Maximum Flexural Strength } \\
(\mathrm{MPa})\end{array}$} \\
Samples & \multicolumn{2}{c}{ Area $(\mathrm{MPa} \mathrm{mm})$} & \multicolumn{2}{c}{$48 \pm 2$} \\
Graphite_A & $3.5 \pm 0.5$ & $3.9 \pm 0.4$ & $45 \pm 3$ & \\
Graphite_B & $4.1 \pm 0.5$ & & $49 \pm 4$ & \\
Graphite_C & $4.2 \pm 0.5$ & & $49 \pm 3$ & $126 \pm 3$ \\
2DLSI_A & $10.0 \pm 1$ & $9.9 \pm 0.4$ & $123 \pm 14$ & \\
2DLSI_B & $9.8 \pm 1.5$ & & $129 \pm 20$ & \\
2DLSI_C & $9.3 \pm 1$ & & $124 \pm 10$ & \\
2DLSI_D & $10.3 \pm 1$ & & $129 \pm 9$ & \\
1DPI_A & $14.8 \pm 1$ & $16.6 \pm 2.9$ & $89 \pm 6$ & \\
1DPI_B & $15 \pm 1$ & & $84 \pm 5$ & \\
1DPI_C & $20 \pm 1.3$ & & $59 \pm 4$ & \\
\hline
\end{tabular}

calculated, for the four 2DLSI samples, the three graphite samples and the three 1DPI samples. It is clear that the calculated area for graphite, 2DLSI and 1DPI are different from one another. The data show that the bidirectional 2DLSI C/C-SiC is a moderately tough material sitting somewhere between brittle graphite and the slightly tougher unidirectional 1DPI C/C-SiC. Although the total number of samples was small, Table 1 indicates that there were significant differences between the three sample types (graphite, 2DLSI, and 1DPI). In addition to estimating the effective toughness of the samples, the fiber architecture can also be examined in the context of potential toughening mechanisms (Flinn \& Trojan, 1990). In the unidirectional 1DPI sample, which exhibits higher toughness, all fibers are oriented parallel to the tensile direction such that more energy is required to deflect the crack. On the other hand, the 2DLSI sample had fewer fibers contributing to crack deflection resulting in a reduced toughness.

Figure 4 compares the failure evolution of three different types of sample, i.e. 2DLSI, 1DPI, and graphite. The reduced toughness of the 2DLSI samples compared with the 1DPI samples appears to be associated with fracture of the pullout fiber bundles close to the crack plane (Fig. 4c). This means that there is only a short tongue of fibers that needs to be dragged a small distance through the surrounding matrix before working free completely. Thus, only a relatively small amount of energy is required to pull out these short fiber tongues, resulting in only a modest effective toughness for the material. In comparison, for the 1DPI_B sample there was no distinct crack observed across the sample throughout the majority of the four-point bend testing. Eventually a crack did appear, but with many fibers still bridging the gap (Fig. 4f). A number of these fibers ended up breaking at locations far from the initial site of the crack. In this case, energy is continually consumed in pulling the fibers out from the surrounding matrix. Finally, graphite as a monolithic ceramic exhibited a single straight crack running through the whole sample (Fig. 4i). This clearly indicates that graphite is not as tough as either of the two CMC samples. 

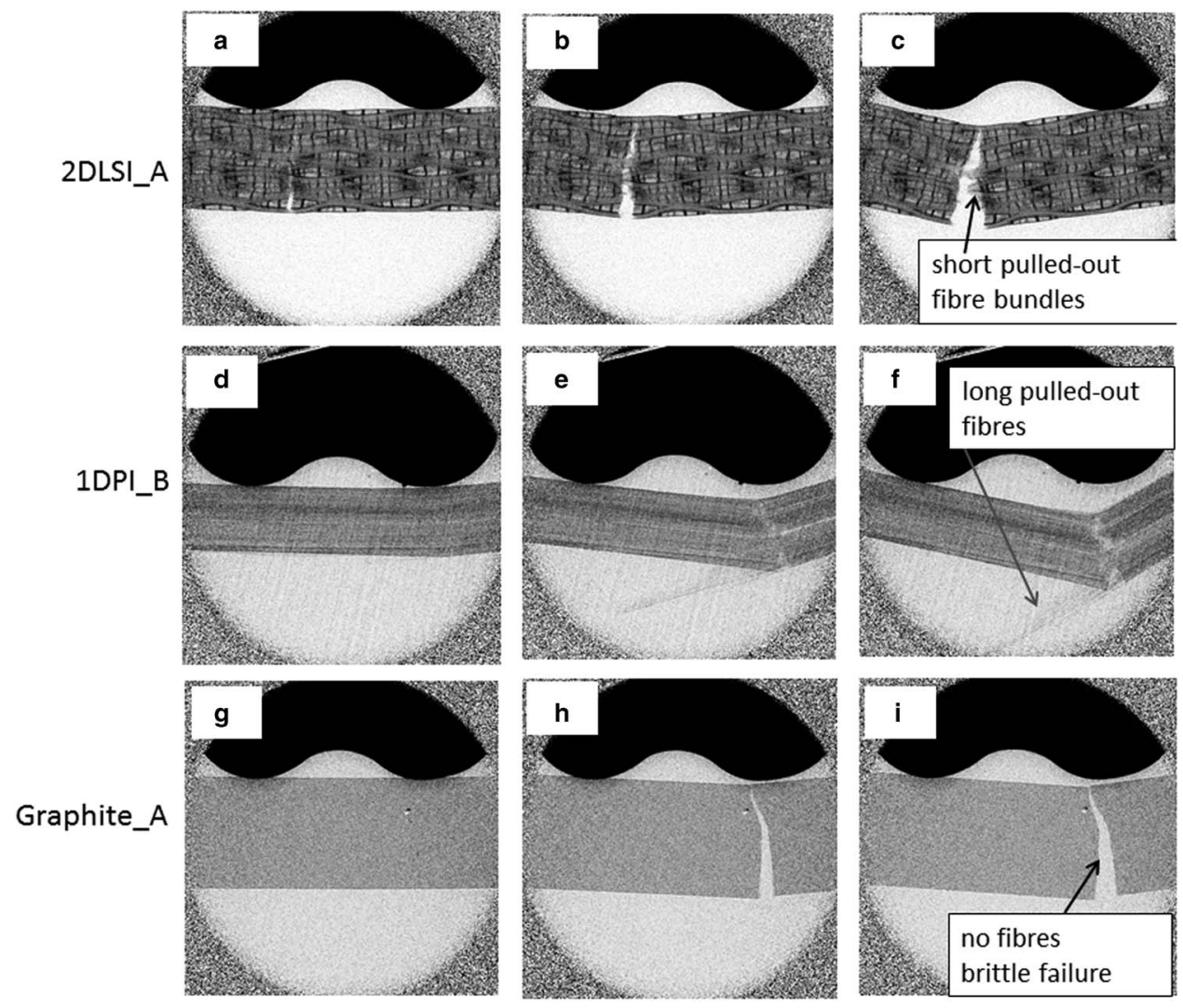

Figure 4. Failure evolution of 2DLSI_A (a-c), 1DPI_B (d-f), and Graphite_A (g-i). The pulled out fiber bundle is short in the 2DLSI sample (b and $\mathbf{c}$ ) and the pulled out fibers length is long in the unidirectional 1DPI sample (e and $\mathbf{f}$ ).

\section{$\mu \mathrm{XCT}$}

$\mu \mathrm{XCT}$ was used to assess the structure of the 2DLSI_A sample after failure. The result shown in Figure 5 illustrates the typical microstructure of the $\mathrm{C} / \mathrm{C}-\mathrm{SiC} \mathrm{CMC}$. The two directionally orientated fiber tows (dark gray) are clearly visible in the XZ plane of Figure $5 \mathrm{~b}$ as is the network of SiC that surrounds each tow (white). Figure $5 b$ shows both the tensile load and crack propagation direction. This view in the XZ sample plane shows how tows of fibers parallel to the tensile load at the crack tip are able to deflect the crack. In contrast, fibers aligned perpendicular to the tensile load have pulled apart and allowed the crack to propagate through undeflected. The spatial resolution of the 3D image was sufficient to detect the presence of discontinuities within individual fibers, as shown in Figures $5 c$ and $5 d$. Very fine scale secondary cracks, which propagated in the same direction as the main crack, are just visible in Figure 5e, as indicated by the small white arrows. The secondary cracks mainly occur within the matrix or at the interface between the matrix and the fibers. It is interesting to note that after testing, the broken parts of the sample were still held together by one tow of fibers running in the horizontal direction across the image (Fig. 5b).

The SiC-rich voxels were determined by volume segmentation of the three-dimensional $\mu \mathrm{XCT}$ reconstructions. The SiC content for the 2DLSI_A sample was found to be $32 \pm 14 \%$ vol. This is higher than the SiC content in the 1DPI_B samples (which is calculated from $\mu \mathrm{XCT}$ data to be $11 \pm 7 \% \mathrm{vol}$ ). The nominal carbon content in 2DLSI_A is $68 \pm 14 \%$ vol and is close to an actual value determined from the manufacturing process (Krenkel, 2008), consisting of both carbon fibers and any residual carbon matrix. Figure 6 shows a 3D rendering of a region of interest (ROI) $\left(\sim 0.5 \times 0.5 \times 1 \mathrm{~mm}^{3}\right)$ within the $\mu \mathrm{XCT}$ reconstruction; this region corresponds to the dotted circle in Figure $5 \mathrm{a}$. The ROI volume was selected as it contained a pulled out fiber bundle spanning the crack after the conclusion of the mechanical four-point bend test. The carbon fibers and carbon matrix are shown in light brown whereas the silicon carbide matrix network is shown in yellow.

After failure, Backscattered imaging in the scanning electron microscope (Fig. 7) was used to image the side of the 2DLSI_A sample in order to confirm the presence and microstructural details of the $\mathrm{SiC}$ and carbon components. Although the electron microscope image is limited to analysis of the near-surface regions, it can produce higher resolution images than X-ray microscopy. This information thus provides a good complement to the $\mathrm{X}$-ray image analysis. The electron microscopy results clearly illustrate that the $\mathrm{SiC}$ is not only confined to the network walls surrounding the fiber bundles, but also forms patches between the individual fibers ("SiC rich" regions in Fig. 7). It should be noted that these 

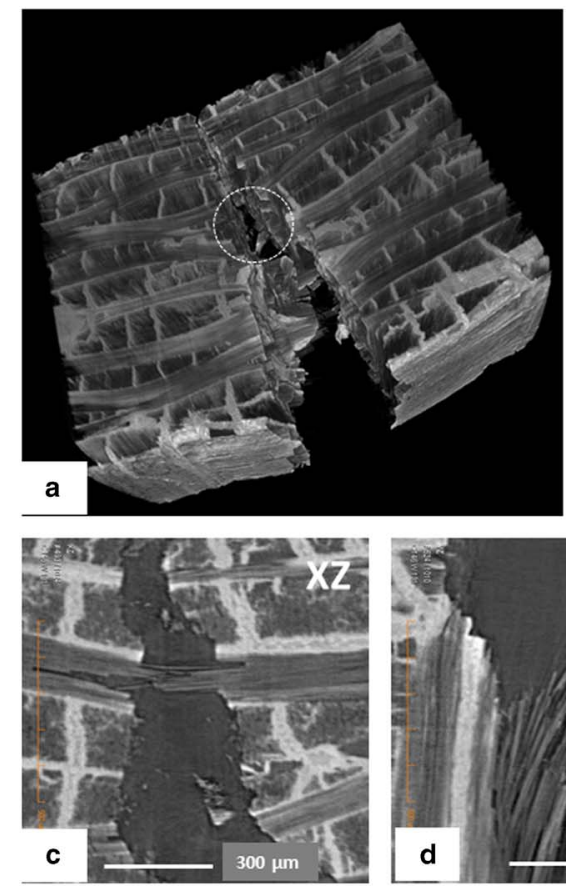
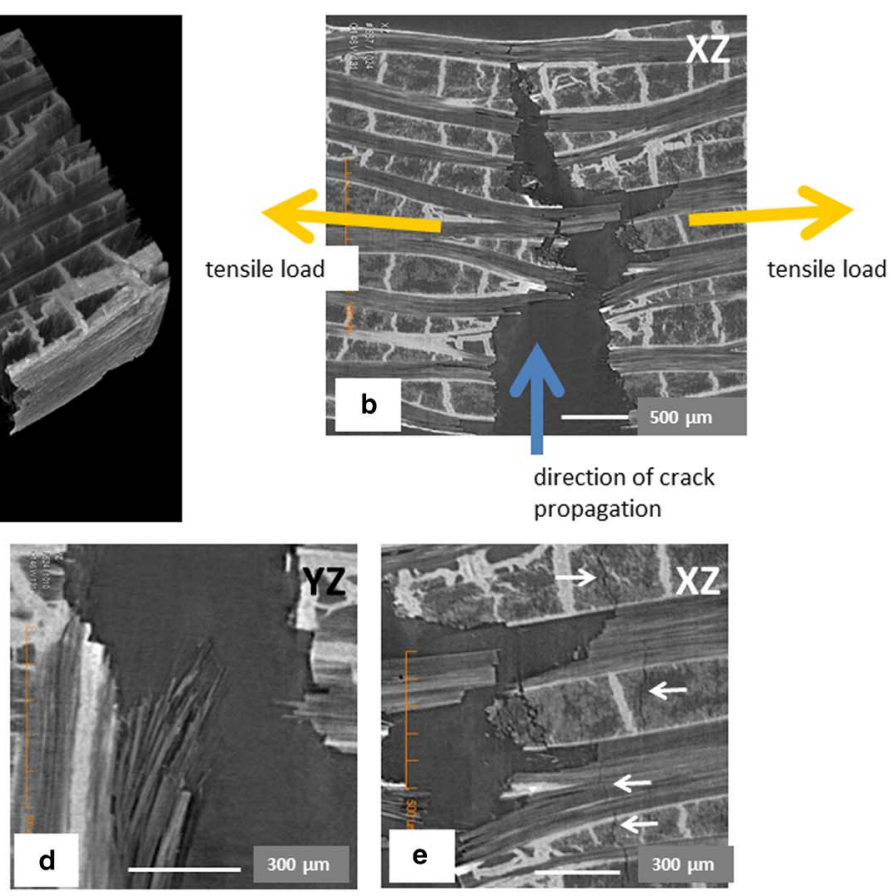

Figure 5. Three-dimensional micro X-ray computed tomography reconstruction of 2DLSI_A sample. a: Threedimensional view. $\mathbf{b}$ : The direction of crack propagation and the local tensile loads on the fiber bundles that once spanned the crack. c: Individual fibers barely visible in XZ plane. d: Broken fiber bundle in YZ plane. e: Very fine secondary cracks (indicated by arrows) that propagate in the same direction as the main crack.

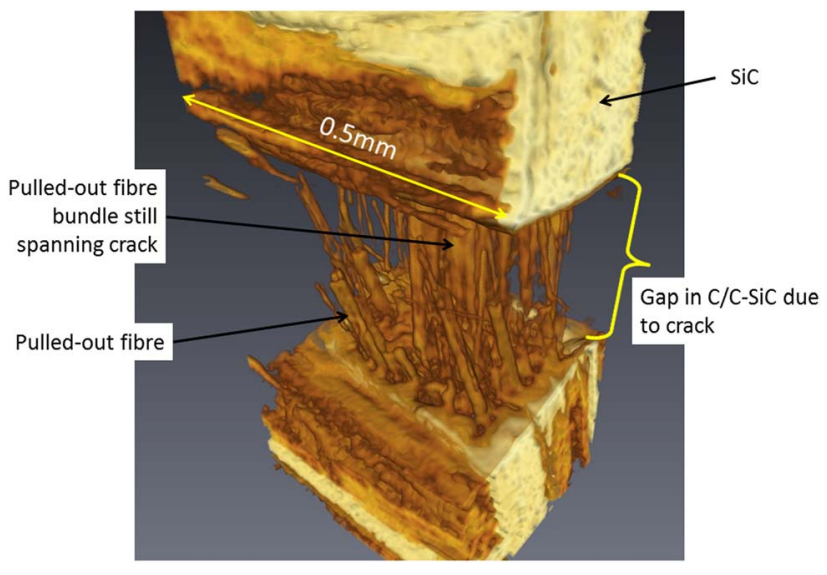

Figure 6. Image rendering of a cropped volume from the dotted circle in Figure 5a of 2DLSI_A sample after failure. It should be noted that this volume was from inside the crack.

patches were not clearly resolved using $\mu \mathrm{XCT}$, due to the polychromatic nature of the source and limited resolution of the system. Moreover, there appears to be a correlation between the fibers that underwent pullout and a low concentration of these SiC patches. The regions of the fiber bundles that are rich in $\mathrm{SiC}$ failed in a brittle manner and did not undergo fiber pullout. To the authors' knowledge, this is the first time that a direct correlation between the strength of $\mathrm{C} / \mathrm{C}-\mathrm{SiC} \mathrm{CMCs}$ and the local microstructure has been observed.

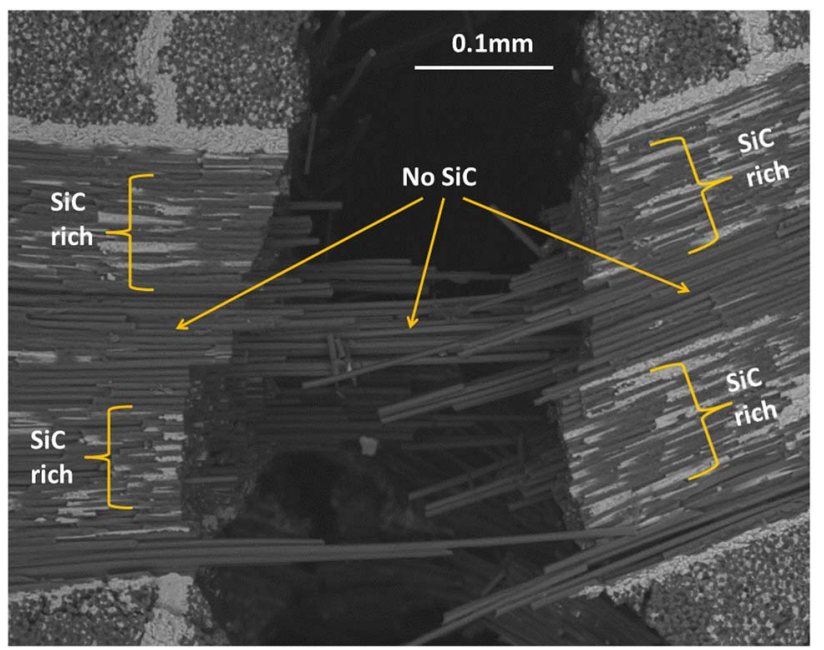

Figure 7. A backscattered electron image showing fiber pullout. In the backscattered electron image, the $\mathrm{SiC}$ appears as a bright gray and the carbon fibers as dark gray; voids are black. It appears that where the fiber bundle is rich in $\mathrm{SiC}$ patches ( $\mathrm{SiC}$ rich) the fracture is brittle with no pullout of fibers, and that fiber pullout is therefore limited to regions of fibers with no $\mathrm{SiC}$ patches.

\section{DISCUSSION}

Radiographic imaging of the $\mathrm{C} / \mathrm{C}-\mathrm{SiC}$ CMC samples during four-point bend testing has enabled us to correlate the shape of the load curve with the local sample microstructure. The correlation of the radiographs to critical points on the 
load/displacement curve shows that the fiber bundles act to slow down crack propagation. This is due to strain energy being consumed in first breaking these fibers and then dragging bundles of fibers out of the surrounding matrix. Minor peaks in the load curve can be directly related to the pullout of bundles of fibers running perpendicular to the plane of the crack. This information is very useful in identifying the microstructural damage characteristics of the material. The subsequent $\mu \mathrm{XCT}$ of the failed sample shows both pullout of fiber bundles and the corresponding gaps in finer detail and in 3D. In the 2DLSI sample, the deflection of the crack out of its average plane and into the tensile load direction causes the overall surface area of the crack to increase. This requires a larger amount of elastic energy than a simple planar crack (Hutchinson \& Jensen, 1990; Krenkel, 2008; Bale et al., 2013) as extra energy is required to break the fibers parallel to tensile load direction and then pull them from the surrounding matrix.

A direct comparison of the radiographic image of the 2DLSI sample with graphite and with 1DPI shows that the 2DLSI sample has moderate toughness. The toughness of the CMC sample was found to depend on the process of "fiber pullout," the fiber direction, the fiber content and the local variations in the $\mathrm{SiC}$ content. Tougher material (1DPI) was found to contain long pulled out fibers with broad load/ displacements curves in the load plot of region III (Fig. 3). When the fiber pullout was short, a sudden drop within region III of the load plot occurred and a reduced toughness was observed. In comparison with the unidirectional 1DPI samples, the fibers in the 2DLSI samples may have broken closer to the site of the crack because they were more highly constrained by the perpendicular fiber tows and the surrounding $\mathrm{SiC}$ matrix. This would likely have prevented the fiber tows from bending to form a large radius of curvature. It may instead have forced them to bend sharply near to the crack. Additional evidence in support of this proposed failure mechanism is given by the SEM results that confirm key features of the X-ray study. In particular, local variations in the $\mathrm{SiC}$ content seem to have a significant influence on the materials strength. The images show that $\mathrm{SiC}$ rich regions within the 2DLSI material could further contribute to brittle fracture behavior, a reduced amount of fiber pullout and reduced material toughness. Increasing the carbon fraction during manufacture before final infiltration with molten silicon, will likely lead to a reduction in the fraction of the $\mathrm{SiC}$ present in the material. This would result in an overall increase in material toughness. A possible mechanism for how this could be achieved, is by increasing the number of polymer re-infiltration-plus-pyrolysis steps, before the final infiltration with molten silicon. However, further testing will be required to assess how the lower $\mathrm{SiC}$ fraction by volume might negatively affect the materials oxidation resistance.

\section{CONCLUSIONS}

The key finding from the present study combining radiography, $\mu \mathrm{XCT}$ and four-point bend testing is that the process of fiber pullout in C/C-SiC CMCs can be linked to the material toughness. Long pulled out fibers, corresponding to broad load/displacements curves within region III of the load plots, indicate a tougher material. Meanwhile, the SEM results show that the local distribution and concentration of $\mathrm{SiC}$ has a critical impact on the material toughness. $\mathrm{SiC}$ is a common choice of additive in CMC manufacture due to its excellent oxidation resistance, however, as our results show, this comes at the cost of reducing the materials toughness. We observe that areas of the material which are rich in $\mathrm{SiC}$ increase bonding between fibers, constricting their movement, and preventing fiber pullout. Regions with less $\mathrm{SiC}$ have much greater flexibility and thus there is a greater capacity for the matrix to absorb strain energy. Previous work has highlighted how $\mathrm{C} / \mathrm{C}-\mathrm{SiC} \mathrm{CMC}$ toughness depends on both the manufacture and composition (Krenkel, 2008). The present study combining load testing and X-ray microscopy highlights how important the local variations in these parameters can be in determining the overall strength of C/C-SiC CMCs.

\section{ACKNOWLEDGMENTS}

The authors acknowledge the support of the Australian Research Council through the Centre of Excellence for Advanced Molecular Imaging. The authors thank to DLR (Institute of Structures and Design, Stuttgart, Germany) for providing some of the samples.

\section{REFERENCES}

Abbey, B., Eve, S., Thuault, A., Charlet, K. \& Korsunsky, A. (2010). Synchrotron X-ray tomographic investigation of internal structure of individual flax fibres. 6th World Congress of Biomechanics (WCB 2010), Singapore, August 1-6, 2010, pp. 1151-1154.

Awaja, F. \& Arhatari, B.D. (2009). X-ray micro computed tomography investigation of accelerated thermal degradation of epoxy resin/glass microsphere syntactic foam. Compos A Appl S 40(8), 1217-1222.

Baimpas, N., Xie, M., Song, X., Hofmann, F., Abbey, B., Marrow, J., Mostafavi, M., Mi, J. \& Korsunsky, A.M. (2014). Rich tomography techniques for the analysis of microstructure and deformation. Int J Comput Method 11(03), 1343006.

Bale, H.A., Haboub, A., MacDowell, A.A., Nasiatka, J.R., Parkinson, D.Y., Cox, B.N., Marshall, D.B. \& Ritchie, R.O. (2013). Real-time quantitative imaging of failure events in materials under load at temperatures above $1,600^{\circ} \mathrm{C}$. Nat Mater 12(1), 40-46.

Budiansky, B. \& Hutchinson, J.W. (1986). Matrix fracture in fiberreinforced ceramics. J Mech Phy Solids 34(2), 167-189.

CZabaj, M.W., Riccio, M.L. \& Whitacre, W.W. (2014). Numerical reconstruction of graphite/epoxy composite microstructure based on sub-micron resolution X-ray computed tomography. Compos Sci Technol 105, 174-182.

Dassios, K.G., Kordatos, E.Z., Aggelis, D.G. \& MatiKas, T.E. (2014). Crack growth monitoring in ceramic matrix composites by combined infrared thermography and acoustic emission. J Am Ceram Soc 97(1), 251-257.

Flinn, R.A. \& Trojan, P.K. (1990). Engineering Materials and Their Applications. New York, USA: John Wiley \& Sons, Inc. 
Frazer, D., Abad, M.D., Krumwiede, D., Back, C.A., Khalifa, H.E., Deck, C.P. \& Hosemann, P. (2015). Localized mechanical property assessment of $\mathrm{SiC} / \mathrm{SiC}$ composite materials. Compos A Appl S 70, 93-101.

Genet, M., Marcin, L., Baranger, E., Cluzel, C., Ladevèze, P. \& Mouret, A. (2012). Computational prediction of the lifetime of self-healing CMC structures. Compos A Appl S 43(2), 294-303.

Heidenreich, B. (2012). Manufacture and applications of C/C-SiC and $\mathrm{C} / \mathrm{SiC}$ composites. In Processing and Properties of Advanced Ceramics and Composites IV, Singh, J.P., Bansal, N.P., Goto, T., Lamon, J., Choi, S.R., Mahmoud, M.M. \& Link, G. (Eds.), pp. 183-198. Hoboken, NJ, USA: John Wiley \& Sons Inc.

Hutchinson, J.W. \& Jensen, H.M. (1990). Model of fiber debonding and pullout in brittle composites with friction. Mech Mater $\mathbf{9}$, 139-163.

KaK, A.C. \& Slaney, M. (1988). Principles of Computerized Tomographic Imaging. New York, NY: IEEE Press.

Korsunsky, A., Hofmann, F., Abbey, B. \& Gaucherin, G. (2010). Synchrotron X-ray analysis of microstructure and microdeformation in a recast AA6063 aluminium alloy. J Strain Anal Eng Des 45(5), 351.

Krenkel, W. (2008). Ceramic Matrix Composites. Weinheim: Wiley-VCH.

Krenkel, W., Heidenreich, B. \& Renz, R. (2002). C/C-SiC composites for advanced friction systems. Adv Eng Mater 4(7), $427-436$.
Nouri, H., Lubineau, G. \& Traudes, D. (2013). An experimental investigation of the effect of shear-induced diffuse damage on transverse cracking in carbon-fiber reinforced laminates. Compos Struct 106, 529-536.

PAL, N.R. \& PAL, S.K. (1993). A review on image segmentation techniques. Pattern Recogn 26(9), 1277-1294.

Pedrazzini, S., Galano, M., Audebert, F., Collins, D.M., Hofmann, F., Abbey, B., Korsunsky, A.M., Lieblich, M., Garcia Escorial, A. \& SMith, G.D.W. (2016). Strengthening mechanisms in an Al-Fe-Cr-Ti nano-quasicrystalline alloy and composites. Mater Sci Eng A 672, 175-183.

Raether, F. (2013). Ceramic matrix composites - An alternative for challenging construction tasks. Ceram Appl 1, 45-49.

Roy, J., Chandra, S., DAS, S. \& MaItra, S. (2014). Oxidation behaviour of silicon carbide - A review. Rev Adv Mater Sci 38, 29-39.

Sloof, W.G., Pei, R., McDonald, S.A., Fife, J.L., Shen, L., BoatemaA, L., Farle, A.-S., Yan, K., Zhang, X., van der ZwaAg, S., Lee, P.D. \& Withers, P.J. (2016). Repeated crack healing in MAX-phase ceramics revealed by $4 \mathrm{D}$ in situ synchrotron X-ray tomographic microscopy. Sci Rep 6, 23040.

Zhu, D., Mobasher, B. \& Rajan, S.D. (2012). Non-contacting strain measurement for cement-based composites in dynamic tensile testing. Cement Concrete Compos 34, 147-155.

Zou, W., Hunter, N. \& Swain, M.V. (2011). Application of polychromatic $\mu \mathrm{CT}$ for mineral density determination. J Dent Res 90(1), 18-30. 